\title{
Evaluación de la Memoria Tipo Episódica en preescolares empleando una tarea con perspectiva egocéntrica
}

\author{
Assessment of Episodic Like-Memory in Preschool Children \\ Using an Egocentric Perspective Task
}

\author{
Eneida Strempler-Rubio, Javier Vila, Angélica Alvarado, \& Rosalba Juárez \\ Universidad Nacional Autónoma de México, Ciudad de México, México
}

\begin{abstract}
Resumen: La Memoria Tipo Episódica (MTE) implica la recuperación integrada de elementos mínimos de la memoria episódica: Qué, Dónde y Cuándo (QDC) de un evento personal. Investigaciones sugieren que una tarea adecuada para niños debe realizarse con una perspectiva egocéntrica y ocurrir en un solo ensayo, porque al realizarse con varios ensayos esto implica el uso de información semántica. Se diseñó una tarea con una perspectiva egocéntrica y realizable en un único ensayo de entrenamiento, que manipuló la magnitud de la consecuencia para dos experiencias, así como el intervalo entre entrenamiento y prueba. Niños preescolares, desde una perspectiva egocéntrica, buscaron monedas grandes o pequeñas en tres contenedores y eligieron el contenido preferido tras dos intervalos de tiempo. La elección de los participantes mostró la recuperación integrada del QDC a partir de un solo ensayo de entrenamiento. Además, las elecciones de los preescolares reflejaron sus estrategias de recuperación: la inmediatez o mayor consecuencia. La tarea se basa en las representaciones del participante más que en la capacidad de reporte verbal, lo que hace a esta preparación sensible a la cognición episódica del recuerdo de la MTE en niños.
\end{abstract}

Palabras clave: memoria episódica, perspectiva egocéntrica, niños preescolares, memoria tipo episódica, qué-dónde-cuándo.
Abstract: Episodic Like-Memory (ELM) involves the integrated retrieval of minimum elements of episodic memory: the What, Where and When (WWW) knowledge of a personal event. Research suggests that an appropriate task for children should be done with an egocentric perspective and occur in a single trial, because if several trials take place, it implies the use of semantic information. A task with an egocentric perspective and achievable in a single training trial was designed; this task manipulated the magnitude of the consequence for two experiences and the interval between training and test. Preschoolers, from an egocentric perspective, searched for big or small coins in three containers and chose their favorite content after two time intervals. The participants' choices showed the integrated retrieval of WWW from a single training trial. In addition, preschoolers' choices reflected their retrieval strategies: immediacy or greater consequence. The task is based on the representations of the participant rather than verbal reporting capability, which makes this preparation sensitive to episodic cognition of ELM recollection in children.

Keywords: episodic memory, egocentric perspective, preschoolers, episodic like-memory, WhatWhere-When.

Esta investigación se realizó con financiamiento del proyecto Papiit IN 301315 subvencionado por la DGAPA, UNAM. La primera autora cuenta con una beca doctoral financiada por Conacyt. Agradecemos las facilidades para llevar a cabo la presente investigación a las estancias infantiles: Alan's y Nuevo Amanecer del Estado de México.

Contacto: J. Vila. División de Investigación y Posgrado, Facultad de Estudios Superiores Iztacala, Universidad Nacional Autónoma de México. Tlalnepantla, Estado de México 54096, México. Correo electrónico: javila@campus.iztacala.unam.mx

Cómo citar: Strempler-Rubio, E., Vila, J., Alvarado, A., \& Juárez, R. (2015). Evaluación de la Memoria Tipo Episódica en preescolares empleando una tarea con perspectiva egocéntrica. Revista de Psicología, 24(2), 1-13. http://dx.doi.org/10.5354/0719-0581.2015.37650 


\section{Introducción}

Originalmente la Memoria Episódica (ME) fue definida como la capacidad de codificar, almacenar y recuperar eventos personales únicos, que cuentan con relaciones espaciales y temporales entre sí que le son significativas al individuo. $\mathrm{La}$ memoria episódica permite acceder al "Qué", "Dónde" y "Cuándo" (QDC) de un determinado evento personal. Esta habilidad se había considerado exclusivamente humana, porque involucra recordar y reexperimentar con noción de sí mismo a través de un "viaje mental en el tiempo" (Tulving, 1972, 1985).

Sin embargo, para averiguar si los animales cuentan con una habilidad similar a la memoria episódica, Clayton y Dickinson (1998) reinterpretaron la definición original retomando aquellos elementos que, a través del comportamiento, exhibieran la capacidad de los animales para recuperar de manera integrada los contenidos del Qué (contenido semántico), el Dónde (contenido espacial) y el Cuándo (contenido temporal) de un evento. Esta forma de memoria fue denominada como Memoria Tipo Episódica (MTE).

En un experimento pionero de la MTE (Clayton \& Dickinson, 1998), una especie de los arrendajos (Aphelocoma coerulescens) almacenaron y recuperaron gusanos (alimento preferido, pero perecedero) y cacahuates (alimento no perecedero, pero de menor preferencia) de dos lados diferentes de una bandeja y a diferentes intervalos de tiempo. Los elementos del evento que conformaron el Qué fue el alimento (gusanos y cacahuates), el Dónde, alguno de los lados de la bandeja y el Cuándo, el valor del intervalo de tiempo. Si la recuperación se realizaba tras un intervalo de tiempo largo, los gusanos eran degradados en sabor y los arrendajos preferían los cacahuates; no obstante, tras un intervalo corto los arrendajos preferían los gusanos, porque aún permanecían frescos. De acuerdo con los autores, estos resultados sugieren que los arrendajos fueron capaces de recordar de manera integrada el Qué, Dónde y Cuándo de un evento. Para demostrar este tipo de memoria en otras especies las tareas diseñadas han sido adaptadas a cada especie: las preparaciones fueron adecuadas a las características ecológicas, sociales y alimentarias del organismo al que se busca atribuir la MTE. Por ejemplo, los estudios con ratas aprovecharon la importancia que tienen los olores y sabores en situaciones naturales (Babb \& Crystal, 2006). En el caso de los estudios con humanos algunas investigaciones se han realizado con niños, porque aún no han desarrollado completamente el lenguaje verbal, lo que permite hacer una comparación entre especies. Además, la MTE puede ser considerada como un antecedente en el desarrollo de la memoria episódica. Si bien existen diversas preparaciones con niños, ninguna ha podido observar la recuperación integrada del QDC así como su ocurrencia a partir de una perspectiva egocéntrica, es decir, que los objetos y acciones tengan ubicaciones espaciales en relación con la experiencia del participante (Russell \& Hanna, 2012).

En el caso de los niños, por ejemplo, Russell, Alexis y Clayton (2010) realizaron un experimento en el que niños de 3,4 y 5 años tuvieron la oportunidad de escoger objetos que usarían para jugar al siguiente día. Los niños de 3 años fueron incapaces de completar la tarea, los niños de 5 años realizaron la tarea en un nivel de tendencia y los niños de 4 años actuaron pobremente. Los autores concluyeron que los niños actuaron funcionalmente, pero no episódicamente. En otro estudio con niños, Russell, Cheke, Clayton y Meltzoff 
(2011) utilizaron una tarea que involucró chocolate (comida preferida) y panecillos (comida menos preferida). El chocolate se derretía luego de un intervalo largo en una caja caliente, mientras los panecillos permanecían intactos en ambas cajas. En este caso, los participantes prefirieron el chocolate en ambas cajas, lo que llevó a suponer que los resultados no se debían a la MTE, sino al gusto por el chocolate.

Por su parte, Hayne e Imuta (2011) entrenaron a participantes de 3 y 4 años de edad a esconder juguetes en posiciones separadas dentro de sus casas. Los juguetes escondidos fueron considerados el Qué; el orden de escondite fue considerado el Cuándo; y el lugar de escondite fue considerado el Dónde. En ambas edades la recuperación del orden fue insuficiente. En una prueba de retentiva verbal, los niños de 4 años de edad proporcionaron más información que los niños de 3 años; mientras que en una prueba de retentiva conductual, los niños de 3 años recuperaron de forma similar a los niños de 4 años. No obstante, la recuperación del orden fue inferior en los niños de 3 años, e insuficiente para ambas edades. Por lo que los autores supusieron que a la edad de 3 años, los niños exhiben habilidades episódicas rudimentarias de memoria, y esa confianza estricta de la retentiva verbal puede menospreciar su habilidad de presentar los contenidos de la MTE. En otro estudio, Russell y Davies (2012) realizaron un experimento con niños de diferentes rangos de edad (18-23, 24-35 y 3647 meses). Los participantes tenían que realizar una secuencia de acciones para que un juguete funcionara: el juguete desempeñó el papel del Qué, y los lados de las palancas en el juguete fueron el Dónde, mientras que el orden de las acciones para que funcionara fue el Cuándo. $\mathrm{Si}$ bien hubo diferencias en las actuaciones de los niños, los autores sugieren que esta preparación es solo un indicador de alguna de las características (e.g. la manera de considerar el Cuándo) a considerar en una tarea de la MTE.

El estudio de la MTE debe implicar una metodología que pueda dar cuenta de la recuperación integrada de los contenidos mínimos (i.e., QDC). Adicionalmente, Rusell y Hanna (2012) proponen que al investigar acerca de este tipo de memoria en niños, es necesario aproximarse desde un punto de vista egocéntrico, es decir, que las conexiones espacio-temporales de un episodio tengan una relación unificada con el niño. Dicha característica puede ser considerada en las tareas con niños, porque estos desde los 18 meses son conscientes de sí como entidad física y tienen noción de sí mismos ubicados en un lugar de acuerdo con una disposición espacial (Gómez, 2007).

Aunque las tareas revisadas en humanos cumplen con la característica del QDC, no han demostrado la recuperación integrada; quizá porque, en gran medida, las pruebas se basan en la competencia verbal del participante. Este sesgo hacia la habilidad verbal hace necesarias pruebas comportamentales que den cuenta de la cognición episódica tanto en aquellos con el lenguaje menos desarrollado (Cheke \& Clayton, 2013), como en aquellos que lo han desarrollado por completo.

A partir de algunos hallazgos recientes es posible aportar información acerca del estudio de MTE y desde esta evidencia explicar la recuperación integrada del QDC con una preparación que no se base en la habilidad verbal del participante. Por ejemplo, Alvarado, Juárez, Cabrera, Strempler y Vila (2012) diseñaron una tarea de búsqueda-encuentra que manipuló el paso del tiempo y la magnitud de las consecuencias, como una manera de apro- 
ximarse al estudio de la MTE en niños preescolares. Los participantes de 4 y 5 años de edad buscaron planillas de estampas en tres contenedores. El Qué fue considerado como muchas o pocas planillas de estampas; el Dónde los tres contenedores; y el Cuándo fue el momento de la elección de planillas de estampas, tras un pasado reciente o distante. A los participantes se les solicitó buscar las planillas de estampas con las que deseaban quedarse. Los resultados mostraron que los niños recuperaron de manera integrada el QDC, sin necesidad de hacer un reporte verbal del contenido o el lugar. Este experimento empleó una tarea de búsqueda-encuentra proveniente de un modelo de forrajeo animal, la regla de ponderación temporal (Devenport \& Devenport, 1994). Para resolver una tarea típica de este modelo los organismos recurren a fuentes de información espaciotemporales, así como a la integración de experiencias sobre la base de su distancia temporal relativa al momento del recuerdo y a la magnitud de la consecuencia obtenida (Devenport \& Devenport, 1994). Así, esta preparación evidencia que los participantes pueden recuperar de manera integrada del QDC de un evento y, al mismo tiempo, que hacen uso de una perspectiva egocéntrica. Esto último porque la elección del beneficio se realiza a partir de las representaciones espacio-temporales que el niño tiene de un episodio en relación con sí mismo. Debido a las características de esta preparación se podría proponer como una tarea factible para el estudio experimental de la MTE en niños preescolares. Sin embargo, la información que aprendieron los participantes durante la tarea pudo ser de tipo semántico, porque los niños fueron entrenados con múltiples ensayos. En consecuencia, para que una tarea sea considerada de carácter episódico requiere que el evento ocurra en un único ensayo.
Por tanto, el objetivo del presente estudio fue diseñar una tarea experimental para el estudio de la MTE en niños. La propuesta debe cumplir con la recuperación integrada del QDC de un evento a partir de un solo ensayo de entrenamiento, al mismo tiempo que requiera del participante usar una perspectiva egocéntrica para realizar la tarea. Para cumplir este objetivo se adaptó la tarea de búsqueda-encuentra utilizada por Alvarado et al. (2012). La tarea empleada manipuló la magnitud de la consecuencia de cada contenedor y el valor del intervalo de tiempo entre el entrenamiento y la prueba de elección. En este experimento se consideró como medida de análisis la elección conductual por uno de los dos contenedores, los niños señalaron entre los contenedores aquel que tenía las monedas preferidas. El análisis a partir de la elección permite evidenciar conductualmente en los participantes la capacidad de recuerdo de la información QDC, independientemente de su capacidad verbal para reportar hechos.

El presente experimento buscó aproximarse al estudio de la memoria en niños, por lo que se solicitó la participación de niños de 4 años de edad, quienes de acuerdo con Russell y Hanna (2012) cuentan con cognición mayormente basada en representaciones que en conceptualizaciones. En específico, los niños de 4 años son capaces de realizar oraciones cortas y simples, así como narrar eventos pasados y hablar del futuro (Zeledón, 2004), pero su lenguaje es de carácter pragmático (Hirsh-Pasek \& Golinkoff, 2005). Además, aunque estos niños reducen sus errores de articulación y han adquirido los mecanismos básicos del sistema de lenguaje, el desarrollo de este no ha concluido y seguirá perfeccionándose hasta llegar al discurso complejo (Gillam \& Gorman, 2004). 
La tarea empleada en el presente experimento recurre a la sucesión de eventos, ya que es una de las formas de más fácil acceso para la representación del tiempo en niños. La sucesión se construye como percepciones que no requieren ser conceptualizadas, sino representadas como una experiencia perceptiva (Russell \& Hanna, 2012). Desde esta idea es más fácil para los niños tener acceso a secuencias perceptivas que se representan; por ello, este experimento divide el evento único en experiencias: experiencia $\mathrm{A}$ (contenedor A) seguida de la B (contenedor B) y cada experiencia cuenta con igual $(\mathrm{A}++++\mathrm{y} \mathrm{B}++++)$ o diferente magnitud de consecuencia $(\mathrm{A}++++\mathrm{y} \mathrm{B}+)$.

Esta propuesta metodológica se diseñó considerando otras habilidades presentes en los niños de 4 años. Primero, la capacidad para detectar semejanzas y diferencias entre objetos, es decir la habilidad de discriminación (Pearce, 2008). Segundo, la capacidad para percibir el tiempo, ya que desde los 3 años los niños poseen principios subyacentes al razonamiento causal que les permite aprender dónde, cuándo y cómo aplicar las reglas de razonamiento causal (Bullock, Gelman, \& Baillargeon, 1982). Además, los niños desde el periodo sensorio motor dominan las seriaciones y discriminan perceptivamente, imitan y posteriormente recuerdan una sucesión de cambios (Piaget, 1926). Tercero, ya que la magnitud de la consecuencia que se manipula en esta tarea está dada por monedas grandes que valen más y monedas pequeñas que valen poco, se consideró la capacidad que tienen los niños para comprender el valor y el uso del dinero durante un acto individual de compra-venta (Berti \& Bombi, 1981). Finalmente, para asegurar que los niños fueran capaces de diferenciar entre lo que se determinó como magnitud de la consecuencia, la tarea considera la capacidad que tienen los niños desde los 12 meses para elegir entre más y menos a partir del tamaño, superficie o volumen (Feigenson, Carey, \& Hauser, 2002). Esta última habilidad permite a los niños que participaron diferenciar desde el tamaño y no necesariamente sobre la base de la denominación de las monedas.

\section{Método}

\section{Participantes}

Participaron 40 niños de 4 años de edad y sin experiencia en la tarea. Todos ellos asistían a estancias infantiles del Estado de México. Los participantes fueron asignados de manera aleatoria a cuatro grupos: $\mathrm{A}=\mathrm{B}$ $0 \mathrm{~h}, \mathrm{~A}=\mathrm{B} 24 \mathrm{~h}, \mathrm{~A}>\mathrm{B} 0 \mathrm{~h}$ y A > B $24 \mathrm{~h}$. La colaboración de los niños fue voluntaria e informada a los padres y tutores de acuerdo con los requerimientos éticos para la investigación con humanos, señalado en el Código ético del psicólogo de la Sociedad Mexicana de Psicología (2009). Las experimentadoras, a través de una solicitud de práctica de investigación expedida por la coordinación de posgrado y la jefatura de la carrera de Psicología de la Facultad de Estudios Superiores Iztacala de la UNAM, solicitaron a las directoras de las estancias acceso a las mismas para realizar el experimento. La solicitud de práctica indicó los horarios de entrada y salida de la estancia, y fue anexada una copia de las identificaciones institucionales de las experimentadoras así como una explicación del experimento por escrito y verbal, para el caso de las profesoras. Las experimentadoras se presentaron con los niños y explicaron que jugarían un juego y tendrían que poner mucha atención, también explicaron que el juego sería individual. Todos los niños recibieron una planilla de estampas al final del experimento, incluso aquellos que no desearon participar o se encontraron fuera del rango de edad. Se presentó un informe de los resul- 
tados obtenidos a las directoras de las estancias infantiles.

\section{Materiales}

Tres contenedores de color amarillo canario con diferentes formas que jugaron los roles de las experiencias A, B y C: una bolsa de regalo de cartoncillo $(9,5 \mathrm{~cm} \times 3,5 \mathrm{~cm} \times 7,0$ $\mathrm{cm})$, una caja de cartón $(6,0 \mathrm{~cm} \mathrm{x} \mathrm{4,0} \mathrm{cm} \mathrm{x}$ $1,6 \mathrm{~cm})$ y un recipiente de plástico en forma de huevo $(3,0 \mathrm{~cm}$ de diámetro y 4,5 cm). Cuatro monedas pequeñas, de un peso mexicano (equivalente a \$0,05947 dólares americanos) y cuatro monedas grandes, de cinco pesos mexicanos (equivalentes a 0,29736 dólares americanos). Una mesa pequeña de aproximadamente $55 \mathrm{~cm}$ de altura y de $55 \mathrm{~cm}$ x $60 \mathrm{~cm}$ de superficie.

\section{Procedimiento}

El experimento fue realizado en un salón de clases dentro de cada estancia infantil. Las medidas de los salones fueron de $4 \mathrm{x}$ $3 \mathrm{~m}$ aproximadamente. Todos los espacios contaron con el mínimo de mobiliario, iluminación y ventilación adecuada. Los contenedores estuvieron equidistantes entre ellos $(10 \mathrm{~cm})$.

La tarea consistió en encontrar monedas, ya fueran pequeñas (un peso mexicano) o grandes (cinco pesos mexicanos) en tres contenedores de igual color y diferente forma que estaban ubicados en una mesa pequeña. El procedimiento constó de dos fases de entrenamiento y una prueba. Cada participante realizó la tarea individualmente. Durante el único ensayo cada contenedor (A, B, y C) fue destapado y tapado para buscar en el siguiente, visitando así los tres contenedores. Durante la prueba se les solicitaba a los participantes elegir el contenedor donde se encontraba el dinero con el que quisieran quedarse, para lo cual señalaban el contenedor.
Los grupos fueron definidos por el valor de la magnitud de la consecuencia de las experiencias $\mathrm{A}$ y $\mathrm{B}(\mathrm{A}=\mathrm{B}$ y $\mathrm{A}>\mathrm{B})$ y por el valor de la distancia temporal entre el entrenamiento y la prueba; inmediata ( 0 h) y demorada (24 h).

El ensayo dio inicio cuando uno de los niños entró al salón de clases para visitar cada uno de los contenedores conforme la experimentadora le indicó. Para garantizar que los participantes conocían la funcionalidad de las monedas, a cada uno de los participantes se les preguntó si sabían su uso; si contestaban de manera negativa, se les informaba que las monedas servían para comprar cosas.

El diseño experimental se presenta en la tabla 1. Si los participantes pertenecían al grupo $\mathrm{A}=\mathrm{B} 0 \mathrm{~h}$, tenían la oportunidad de encontrar cuatro monedas de igual tamaño en cada fase (monedas grandes en $\mathrm{A}$ y $\mathrm{B}$, o monedas pequeñas en $\mathrm{A}$ y B). Los niños debían buscar monedas en cada uno de los contenedores. El contenedor $\mathrm{C}$ nunca tuvo monedas. Cada contenedor era destapado y tapado antes de continuar la búsqueda en el siguiente. Durante la primera fase solo el contenedor A almacenó monedas. Una vez que el niño encontraba las monedas en el contenedor A, la experimentadora le pedía que las tocara, las contara y dijera si eran grandes o pequeñas, si respondían de manera equivocada se les corregía. También se le preguntó al participante si sabía qué tanto se podía comprar con aquellas monedas; si los participantes contestaban de manera incorrecta (e.g. "con monedas grandes se puede comprar poco o con pequeñas se puede comprar mucho") se les corregía diciendo: "con monedas grandes, mucho; y con pequeñas, poco". A continuación, la experimentadora pedía al participante que saliera un instante del salón. 
Tabla 1

Diseño experimental

\begin{tabular}{lcccc}
\hline Grupo & Fase 1 & Fase 2 & IR & Prueba \\
\hline A = B 0 h & A+/B-/C- & A-/B+/C- & $0 \mathrm{~h}$ & \\
A = B 24 h & A+/B-/C- & A-/B+/C- & $24 \mathrm{~h}$ & \multirow{2}{*}{ ¿A, B, C? } \\
\cline { 1 - 4 } A > B 0 h & A++++/B-/C- & A-/B+/C- & $0 \mathrm{~h}$ & \\
A > B 24 h & A++++/B-/C- & A-/B+/C- & $24 \mathrm{~h}$ & \\
\hline
\end{tabular}

Nota: Dos tipos de prueba: inmediata $(0 \mathrm{~h})$ o demorada $(24 \mathrm{~h})$. El valor relativo de la experiencia se representa por: + representa cuatro monedas de $\$ 1 \mathrm{y}++++$ representa cuatro monedas de $\$ 5$. La prueba consistió en un solo ensayo y sin monedas. A, B y C corresponde a los contenedores.

La segunda fase dio inicio cuando se le pedía al participante ingresara de nuevo al salón y se ubicara en la mesa donde se estuvo llevando a cabo la tarea. El procedimiento fue similar a la primera fase, solo que únicamente el contenedor B tuvo monedas de igual tamaño a las encontradas en la fase anterior. Después de la segunda fase de entrenamiento la experimentadora volvía a solicitar al participante que saliera y esperara afuera por unos instantes. Tanto en la primera fase (monedas en el contenedor A), como en la segunda fase (monedas en el contenedor $\mathrm{B})$, el contenedor $\mathrm{C}$ nunca tuvo monedas.

La fase de prueba inició cuando el participante entraba de nuevo al salón y se ubicaba en la mesa donde se llevó a cabo la tarea en las fases anteriores. La prueba consistió en ubicar los tres contenedores sin monedas sobre la mesa, mientras la experimentadora pedía al participante que entrara y le decía: "Busca las monedas con las que te quieres quedar". La experimentadora registraba la respuesta de elección del participante ante dicha solicitud.

Para asegurar que la preferencia fuera por el contenido y no por el contenedor, cada forma del recipiente se contrabalanceó entre los participantes durante el entre- namiento y la fase de prueba, de tal manera que la forma del contenedor A y B cambió entre los participantes. Por ejemplo, mientras para un participante el contenedor A era la bolsa de cartoncillo, para otro participante el contenedor A fue el recipiente en forma de huevo.

El procedimiento de entrenamiento y prueba para los participantes del grupo $\mathrm{A}=\mathrm{B}$ $24 \mathrm{~h}$ fue similar al descrito para el grupo A $=\mathrm{B} 0 \mathrm{~h}$, solo que la fase de prueba se realizó tras $24 \mathrm{~h}$ del entrenamiento.

El procedimiento fue similar para los grupos A > B $0 \mathrm{~h}$ y A > B $24 \mathrm{~h}$, con la diferencia que el contenedor A en la primera fase almacenó cuatro monedas de mayor tamaño (\$5). Para el grupo A > B 0 h la prueba se realizó de manera inmediata tras el entrenamiento (prueba reciente), mientras que para el grupo A > B $24 \mathrm{~h}$ la prueba se realizó tras $24 \mathrm{~h}$ del entrenamiento (prueba demorada).

\section{Resultados}

Como variable dependiente se consideró la elección a los contenedores que realizaron los participantes durante la prueba. Se hicieron comparaciones entre los grupos. Un ANOVA de 2 (magnitudes de la conse- 
cuencia: $\mathrm{A}>\mathrm{B}$ y $\mathrm{A}=\mathrm{B}$ ) x 2 (intervalos de retención: 0 h y 24 h) reveló efectos principales significativos para ambas variables $[F(3,36)=13,6829, p<, 05$ y $F(3,36)=$ $19.21, p<, 05]$. Aunque el ANOVA reveló efectos significativos para ambas variables independientes, no hubo interacción de estos factores. Tras una Prueba Tukey se encontraron diferencias entre los grupos con prueba inmediata o demorada $(p<$ ,01), $\mathrm{A}=\mathrm{B} 24 \mathrm{~h}$ que difirió con $\mathrm{A}>\mathrm{B} 0 \mathrm{~h}$ $\mathrm{y}$ con $\mathrm{A}=\mathrm{B} 0 \mathrm{~h}, \mathrm{~A}>\mathrm{B} 24 \mathrm{~h}$ que difirió con $\mathrm{A}>\mathrm{B} 0 \mathrm{~h}$ y con $\mathrm{A}=\mathrm{B} 0 \mathrm{~h}$ ); también entre magnitudes de la consecuencia $(p<$ $, 05), \mathrm{A}=\mathrm{B} 24 \mathrm{~h}$ difirió con $\mathrm{A}>\mathrm{B} 24 \mathrm{~h}$. Una $d$ de Cohen reveló que el tamaño del efecto entre las dos diferencias principales fue alto (ver figura 1): 0,63 para la dife- rencia entre $\mathrm{A}>\mathrm{B} 24 \mathrm{~h}$ y $\mathrm{A}=\mathrm{B} 24 \mathrm{~h}, \mathrm{y}$ 0,91 para la diferencia entre A > B $24 \mathrm{~h}$ y A > B $0 \mathrm{~h}$. Este análisis permite evidenciar con mayor certeza que en la elección de los participantes influyó tanto el paso del tiempo, como la magnitud de la consecuencia.

Los datos muestran que los participantes de los grupos A = B 0 h y A > B 0 h eligieron el contenedor $\mathrm{B}$, que es aquel en donde los niños habían encontrado las monedas recientemente. Sin embargo, en el grupo $\mathrm{A}=\mathrm{B} 24 \mathrm{~h}$, los participantes eligieron de igual manera los contenedores $\mathrm{A}$ y $\mathrm{B}$; mientras que en el grupo $\mathrm{A}>$ B 24 h, los participantes eligieron más el contenedor A.

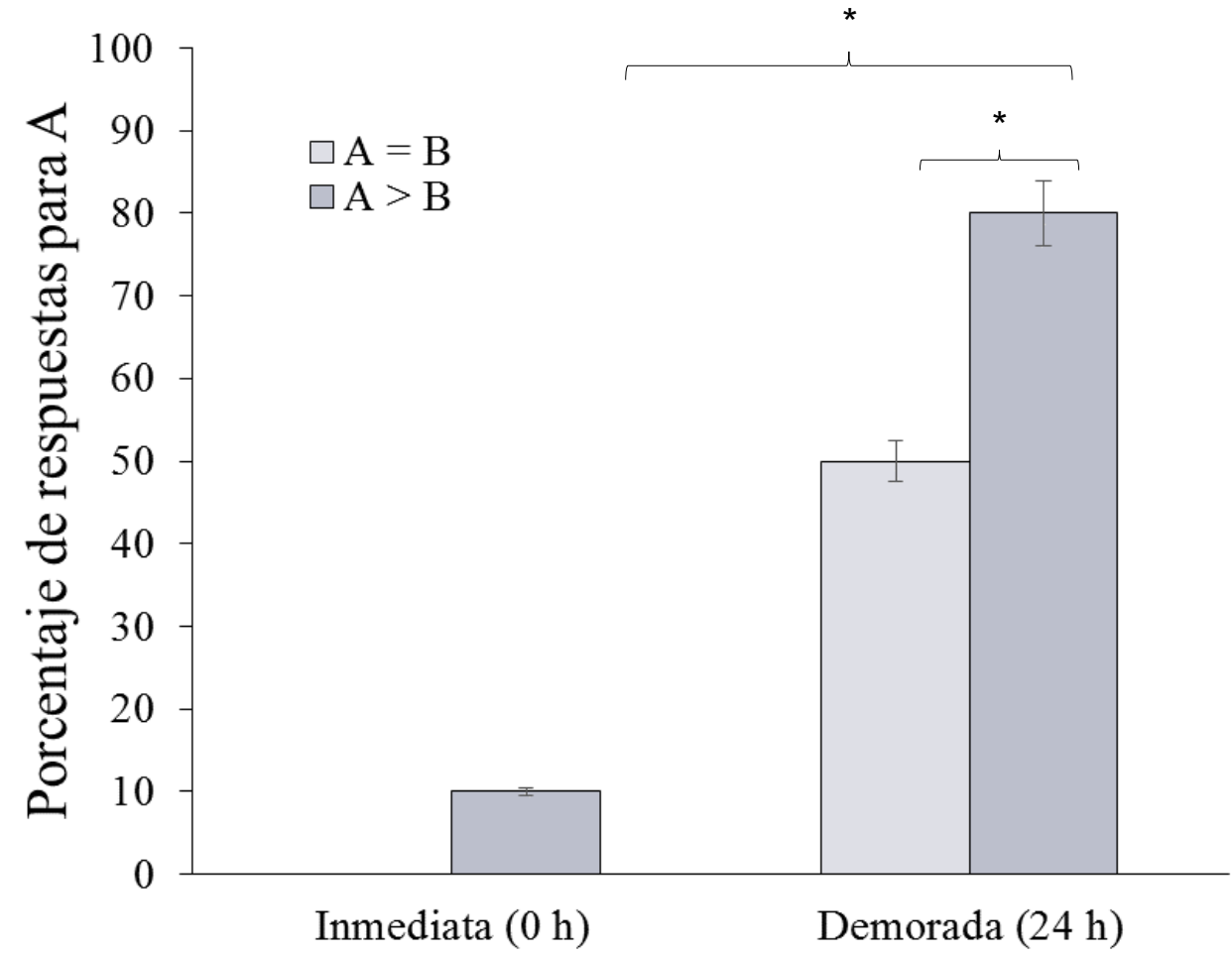

Figura 1. Porcentaje de elecciones al contenedor A. Para los grupos con prueba inmediata $(\mathrm{A}=\mathrm{B} 0 \mathrm{~h}$ y $\mathrm{A}>\mathrm{B} 0 \mathrm{~h})$ el porcentaje de elección del contenedor A fue menor en comparación con los grupos de prueba demorada $(\mathrm{A}=\mathrm{B} 24 \mathrm{~h}$ y A > B $24 \mathrm{~h})$ : preferencia por la opción recientemente reforzada (contenedor $\mathrm{B}$ ). El corchete superior muestra la diferencia significativa entre A > B $0 \mathrm{~h}$ y A > B $24 \mathrm{~h}$, mientras que el corchete inferior muestra la diferencia significativa entre $\mathrm{A}=\mathrm{B} 24 \mathrm{~h}$ y $\mathrm{A}>\mathrm{B} 24 \mathrm{~h}$. 
La diferencia entre los grupos $\mathrm{A}=\mathrm{B} 0 \mathrm{~h}$ y $\mathrm{A}=\mathrm{B} 24 \mathrm{~h}$, con igual magnitud de consecuencia en ambas fases, muestra que al realizar la prueba a las $0 \mathrm{~h}$ ocurre una preferencia por el contenedor recientemente reforzado (B); y que al realizar la prueba a las $24 \mathrm{~h}$ existe indiferencia en la preferencia por ambos contenedores, A y B. La diferencia entre los grupos $\mathrm{A}>\mathrm{B} 0 \mathrm{~h}$ y $\mathrm{A}>\mathrm{B}$ $24 \mathrm{~h}$ muestra que, en los contenedores con diferente magnitud de la consecuencia, si la prueba se realiza a las $0 \mathrm{~h}$ la preferencia es mayor para el contenedor recientemente reforzado (B), pero si esta se realiza a las 24 $\mathrm{h}$ la preferencia es mayor para el contenedor con mayor magnitud de consecuencia (A). Ambas diferencias indican que el intervalo de retención afecta la preferencia al contenedor recientemente reforzado, ya que a las $0 \mathrm{~h}$ se elige el contenedor recientemente reforzado, independientemente de la magnitud de la consecuencia. Sin embargo, la diferencia observada entre los grupos A $=\mathrm{B} 24 \mathrm{~h}$ y A $>$ B $24 \mathrm{~h}$ permite suponer que no es solo dependiente del paso del tiempo, sino también de la magnitud de la consecuencia. En tanto que el análisis de resultados muestra que las elecciones durante la fase de prueba ocurren en función del paso del tiempo y de la diferencia de magnitud de la consecuencia.

\section{Discusión}

Los presentes datos confirman que a partir de la tarea empleada en este estudio es posible observar que los niños desde un ensayo recuperan de manera integrada el QDC de un evento. Siendo el Qué las monedas (pequeñas o las grandes); el Dónde los contenedores; y el Cuándo el momento en que el niño realiza la elección tras el entrenamiento: en un pasado distante o uno reciente.

Además, los resultados obtenidos son similares a los de Alvarado et al. (2012), quienes también manipularon tiempo y magnitud de la consecuencia, pero emplearon más de un ensayo de entrenamiento. Esta similitud en resultados, hace suponer que tanto el tiempo como la magnitud de la consecuencia posibilitan que ambas experiencias se diferencien haciendo una de ellas más significativa que otra. El hecho de que el recuerdo integrado de los participantes suceda de manera similar con uno o más ensayos de entrenamiento sugiere que el empleo de varios ensayos fortalece la consolidación del evento en la memoria semántica (Griffiths, Dickinson, \& Clayton, 1999). Al mismo tiempo, la ocurrencia de la recuperación integrada del QDC a partir de un ensayo señala la necesidad de incorporar esta característica a la definición de la MTE en humanos, por ser comparable a un acontecimiento que se constituye como un evento memorable.

Los resultados obtenidos reflejan las estrategias de recuperación de la MTE en niños de 4 años: inmediatez de la experiencia o magnitud de la consecuencia. En este sentido, el paso del tiempo permite al participante elegir sobre la base de la magnitud de la consecuencia, tal y como puede observarse en la diferencia entre los grupos A > B 24 h y A = B $24 \mathrm{~h}$, la cual se muestra en la figura 2 . En este caso, aunque ambas pruebas se realizan tras $24 \mathrm{~h}$, debido a la diferencia en las magnitudes de la consecuencia la preferencia es mayor en el grupo A > B $24 \mathrm{~h}$. Sin embargo, en los casos donde la elección se realizó de manera reciente a las experiencias, $\mathrm{A}=\mathrm{B} 0 \mathrm{~h}$ y $\mathrm{A}>\mathrm{B} 0 \mathrm{~h}, \mathrm{los}$ niños eligen a partir del beneficio que obtienen de la inmediatez de la consecuencia sin importar la magnitud. Ambas observaciones permiten suponer que el paso del tiempo hace que el participante integre las experiencias para poder compararlas. Así, en el caso de una elección 
reciente el beneficio de la inmediatez hace que la experiencia más próxima temporalmente predomine; mientras que en una elección demorada se disipa el efecto de recencia y se consideran ambas experiencias para elegir aquella de mayor consecuencia. Sin embargo, para indagar más en esta estrategia de elección es necesario considerar otra variación de la magnitud de la consecuencia: $\mathrm{A}<\mathrm{B}$, de tal manera que la inmediatez y la magnitud de la consecuencia apuntan a un mismo sentido. Quizá la ausencia de interacción entre las variables (intervalo de retención y magnitud de la consecuencia) indica la necesidad de esta manipulación; además, dicha manipulación permitiría profundizar en el papel que juega del paso del tiempo como mediador de la elección.

Por otro lado, esta preparación metodológica en comparación con las descritas anteriormente (Hayne \& Imuta, 2011; Russell \& Davies, 2012; Russell et al., 2010; Russell et al., 2011) es sensible a la cognición episódica del recuerdo de la MTE. Por ejemplo, los resultados obtenidos en este experimento reflejan que los participantes actúan episódica y funcionalmente al mismo tiempo, ya que en esta tarea eligen el mejor beneficio a partir de recuperar el QDC de manera integrada desde un único evento. Los resultados también muestran que no hay una preferencia sesgada por un solo elemento, porque los niños diferencian entre la magnitud de consecuencia de cada uno de los contenedores y los momentos en que hacen su elección. Finalmente, la prueba es de carácter conductual y esto posibilita evaluar el recuerdo del participante y no la capacidad de verbalizar ese recuerdo.

A partir de estos resultados es posible suponer que los niños son capaces de previsión, como ocurre en la prueba demorada, ya que tras el paso del tiempo comparan el beneficio que ambas experiencias les aportan, mientras que en la prueba reciente el beneficio que el niño pondera es la inmediatez. Gracias a la previsión los organismos pueden asegurar beneficios futuros y evitar desastres, así como a la capacidad de imaginar eventos futuros para guiar las acciones actuales. Esta habilidad de previsión ha sido documentada incluso en niños pequeños (ver Suddendorf \& Moore, 2011).

Como se mencionó en la introducción, una tarea adecuada para el estudio de la MTE, requiere considerar las características ecológicas, sociales, alimentarias, etc. del animal en el que se estudia la MTE, por lo que proponer una tarea válida para el estudio de la MTE en niños permite la comparación entre especies, de tal manera que se pueda proponer un probable mecanismo común, subyacente en el recuerdo de eventos esenciales para la sobrevivencia del organismo.

En cuanto a la capacidad de recuperación del recuerdo, los niños a partir de sí mismos representan al mundo a través de relaciones espacio-temporales entre objetos y eventos (Russell \& Hanna, 2012). Por ello, la tarea de búsqueda-encuentra asegura una perspectiva egocéntrica, ya que posibilita que los objetos y acciones tengan ubicaciones espaciales en relación con el participante. Los contenedores, monedas y el momento de la elección se encuentran referidos a la percepción del niño, por ejemplo, la búsqueda de las monedas preferidas en los contenedores de acuerdo con el momento de la elección o la interacción con las monedas al tocarlas y contarlas. La tarea se diseñó para que los niños considerasen los beneficios obtenidos, por lo que tiempo y espacio son incorporados en torno a su propia percepción y representación del QDC. Los beneficios de buscar-encontrar y elegir permiten al 
niño incorporarlos y representarlos en torno a sí mismos para tener noción de un evento (Russell \& Hanna, 2012). Por ejemplo, la presente tarea contempla que en la prueba los niños busquen las monedas preferidas, esto asegura que los niños representan el evento a partir de la noción física de sí mismos. Por tanto, la recuperación de información ocurre de manera integrada en torno a la persona: recordar el Qué favorito también representa el Dónde y Cuándo; y al ocurrir en un solo ensayo de entrenamiento el evento, se integra como algo único.

Si pensamos a la MTE como antecedente de la memoria episódica, esta propuesta experimental permite contar con una prueba basada en los contenidos mínimos QDC para acceder al precedente del recuerdo episódico en niños. Debido a que los niños no tienen desarrollado el lenguaje por completo, esta tarea se enfoca en la capacidad de recuerdo independientemente de la capacidad para hablar. De hecho, desde hace dos décadas, la investigación en niños ha demostrado que incluso los menores de 3 años, cuentan con recuerdos acerca de su pasado. Aunque es hasta los 2 años que la memoria a largo plazo es fiable, gracias a las pruebas no verbales se sabe que los niños son capaces de crear recuerdos accesibles, incluso que recuerdan su pasado (Bauer, 2006). Por ello, el presente estudio aplica una prueba de carácter conductual, ya que a partir de la elección del participante es posible inferir lo que está recuperando, aun si son capaces o no de hablar acerca de ello. Asimismo, el estudio de la MTE puede ayudar a identificar cuándo y cómo aparece y se consolida la memoria episódica en los niños.

Los resultados y la tarea aquí expuestos proponen una alternativa metodológica para el estudio de la MTE en niños, ya que se puede mostrar una recuperación integrada del QDC con una tarea realizada con perspectiva egocéntrica y que ocurre a partir de un solo ensayo de entrenamiento. Además, esta preparación permite llevar el estudio de la MTE de animales no-humanos a animales humanos, y con ello suponer la generalidad del fenómeno, posibilitando un análisis comparado con otras especies. Esta generalidad quizá sea una habilidad común respecto de representar el mundo. Por ejemplo, tanto los monos y los niños (desde la edad de un año), representan acontecimientos con objetos y ocurre de manera similar que en humanos adultos, aunque en estos primates el lenguaje verbal no está presente o comienza a desarrollarse en el caso de los niños (e.g., Gómez, 2007). En este experimento se puede observar que los niños son capaces de representar monedas en contendores; también pueden representar que las monedas grandes aportan un mayor beneficio en comparación con las monedas pequeñas. Tal vez la representación a partir de la experiencia con el mundo sea una dimensión que comparten los primates con los humanos. Esto quizá porque existe una capacidad más básica y primitiva en primates, incluido el humano: la capacidad de construir representaciones del mismo tipo o de diferentes tipos a partir de los rasgos distintivos de los objetos. Gracias a los estudios comparados entre niños y otros primates es posible conjeturar que la capacidad para representar el mundo estaba establecida en la evolución de los primates antes de que apareciera el lenguaje humano (Hauser \& Carey, 1998). De continuar con este tipo de estudios comparados, sería posible además de proporcionar indicios de un mecanismo básico compartido, evidenciar la manera cómo este se adapta, modifica y evoluciona en diversas formas. 


\section{Referencias}

Alvarado, A., Juárez, R., Cabrera, R., Strempler, E., \& Vila, J. (2012). Efectos del tiempo y del valor subjetivo de las experiencias en niños preescolares. Acta de Investigación Psicológica, 2, 868-877. Recuperado de http://is.gd/8BP2zn

Babb, J. \& Crystal, J. (2006). Discrimination of what, when, and where is not based on time of day. Learning \& Behavior, 34, 124-130.

http://dx.doi.org/10.1016/j.cub.2006.05.025

Bauer, P. J. (2006). Constructing a past in infancy: A neuro-developmental account. Trends in Cognitive Sciences, 10(4), 175-181.

http://dx.doi.org/10.1016/j.tics.2006.02.0099

Berti, E. \& Bombi, A. (1981). The development of the concept of the money and its value: A longitudinal study. Child Development, 52, 1179-1182.

http://dx.doi.org/10.2307/11295044

Bullock, M., Gelman, R., \& Baillargeon, R. (1982). The development of causal reasoning. En W. J. Friedman (Ed.), The developmental psychology of time (pp. 209-254). New York: Academic Press.

Cheke, L. G. \& Clayton, N. S. (2013). Do different tests of episodic memory produce consistent results in human adults? Learning \& Memory, 20(9), 491-498.

http://dx.doi.org/10.1101/lm.030502.113

Clayton, N. \& Dickinson, A. (1998). Episodic-like memory during cache recovery by scrub jays. Nature, 395, 272-274.

http://dx.doi.org/10.1038/26216

Devenport, L. \& Devenport, J. (1994). Time-dependent averaging of foraging information in least chipmunks and golden-mantled ground squirrels. Animal Behavior, 47, 787-802. http://dx.doi.org/10.1006/anbe.1994.11111

Feigenson, L., Carey, S., \& Hauser, M. (2002). The representations underlying infants' choice of more: Object files versus analog magnitudes. Psychological Science, 13(2), 150-156. http://dx.doi.org/10.1111/1467-9280.004277

Gillam, R. B. \& Gorman, B. K. (2004). Language and discourse contributions to word recognition and text interpretation. En R. B. Gillam \& B. K. Gorman (Eds.), Language and literacy learning in schools (pp. 63-97). New York: Guilford Press.

Gómez, J. (2007). El desarrollo de la mente en los simios, los monos y los niños. Madrid: Morata.

Griffiths, D., Dickinson, A., \& Clayton, N. (1999). Episodic memory: What can animals remember about their past? Trends in Cognitive Sciences, 3(2), 74-80. http://dx.doi.org/10.1016/s1364-6613(98)01272-8

Hayne, H. \& Imuta, K. (2011). Episodic memory in 3- and 4-year-old children. Developmental Psychobiology, 53, 317-322.

http://dx.doi.org/10.1002/dev.20527 
Hirsh-Pasek, K. \& Golinkoff, R. M. (2005). Einstein nunca memorizó, aprendió jugando. Madrid: RM Ediciones.

Hauser, M. D. \& Carey, S. (1998). Building a cognitive creature from a set of primitives: Evolutionary and developmental insights. En D. D. Cummins \& C. Allen (Eds.), The evolution of mind (pp. 51-106). New York: Oxford University Press.

Pearce, J. (2008). Animal learning and cognition: An introduction ( $3^{\text {th }}$ edition). New York: Psychology Press, Taylor.

Piaget, J. (1926). Introducción a la representación del mundo en el niño. Madrid: Morata.

Russell, J., Alexis, D., \& Clayton, N. (2010). Episodic future thinking in 3- to 5-year-old children: The ability to think of what will be needed from a different point of view. Cognition, 114(1), 56-71.

http://dx.doi.org/10.1016/j.cognition.2009.08.013

Russell, J., Cheke, L., Clayton, N., \& Meltzoff, A. (2011). What can What-When-Where (WWW) binding tasks tell us about young children's episodic foresight? Theory and two experiments. Cognitive Development, 26, 356-370.

http://dx.doi.org/10.1016/j.cogdev.2011.09.002

Russell, J. \& Davies, J. (2012). Space and time in episodic memory. En L. Filipović \& K. Jaszczolt (Eds.), Space and time in languages and cultures: Language, culture, and cognition (pp. 283-303). Amsterdam: John Benjamins Publishing Company.

http://dx.doi.org/10.1075/hcp.37

Russell, J. \& Hanna, R. (2012). A minimalist approach to the development of episodic memory. Mind \& Language, 27, 29-54. http://dx.doi.org/10.1111/j.1468-0017.2011.01434.x

Sociedad Mexicana de Psicología. (2009). Código ético del psicólogo. México: Trillas.

Suddendorf, T. \& Moore, C. (2011). Introduction to the special issue: The development of episodic foresight. Cognitive Development, 26(4), 295-298.

http://dx.doi.org/10.1016/j.cogdev.2011.09.001

Tulving, E. (1972). Episodic and semantic memory. En E. Tulving \& W. Donaldson (Eds.), Organization of memory (pp. 381-403). New York: Academic.

Tulving, E. (1985). Memory and consciousness. Canadian Psychology/Psychologie Canadienne, 26(1), 1-12.

http://dx.doi.org/10.1037/h0080017

Zeledón, M. (2004). Lenguaje y estudios sociales en la educación infantil. Costa Rica: EUNED. 